\title{
Relax-Type Magnetometer with Direct Optocoupler Relaxation
}

\author{
P. LipovskÝ*, M. Šmelko, M. Fil’ko, K. Draganová, J. Blažek, J. HudÁk, \\ R. Andoga And S. SzABO \\ Technical University of Košice, Faculty of Aeronautics, Rampová 7, 04121 Košice, Slovak Republic
}

\begin{abstract}
This article deals with the new simplified relax-type magnetometer design, which uses the measurement of the transient effects during time intervals of the sensor after the periodical saturation of the magnetic core in the sensor. The proposed design uses the unipolar power supply for the whole measurement chain and simplified electronics compared to the previous design, from which it was derived. The excitation control, measurement of time intervals, and primary signal processing is carried out by the small FPGA connected to the microcontroller. The initial testing results show that although the presented design is cheap and simple, the achievable sensitivity is better than $5 \mathrm{nT} / \mathrm{LSB}$ and the noise is less than $800 \mathrm{pT} / \sqrt{ } \mathrm{Hz}$ at $10 \mathrm{~Hz}$ in the measurement range $\pm 80 \mu \mathrm{T}$, whereas the sampling frequency is $1000 \mathrm{~Hz}$.
\end{abstract}

DOI: 10.12693/APhysPolA.137.681

PACS/topics: magnetometer, relax-type, optocoupler, microcontroller

\section{Introduction}

The relax-type magnetometers have been the object of development at the Department of Aviation Technical Studies of the Faculty of Aeronautics over many decades. During this time there have been continuous improvements that have led to the laboratory and also industrial applications of the developed magnetometers [1]. However, the development in electronics to the idea of simplifying the magnetometer electronic circuitry, with the possibility of reduction of the printed circuit board dimensions or future further on-chip integration, compared with the previous magnetometer design. The main task was to achieve sufficient parameters for some industrial applications, e.g. navigation devices and detectors on conveyor belts, namely the sensitivity of $5 \mathrm{nT} / \mathrm{LSB}$ or better. The second task was to provide a simple affordable magnetometer that can be used for education in the area of magnetic fields measurement.

\section{Operation principle}

The relax-type magnetometers are a part of the fluxgate magnetometers family, since they also use the magnetic flux gating on the magnetization characteristics of the sensor core [2-4]. However, they do not use the feedback system and instead of the harmonic excitation signal and detection of harmonics, the rectangular pulses excitation signal is used and the duration or other parameters of the transient effects in the relaxation circuits are measured. Since the measurement of the time can be done relatively simply and accurately, the effective operation principle of the relax-type magnetometer is the

*corresponding author; e-mail: pavol.lipovsky@tuke.sk conversion of the magnetic field measurement into the measurement of the time interval differences $[5,6]$.

The magnetic sensor for the relax-type magnetometer consists of two concentric coils - excitation and sensing. As the core of the sensor, several stripes of amorphous ribbons from VITROVAC VAC6025 or VAC6030 are mostly used. Also, experimental materials were tested [7] and currently bulk cores made from magnetic microwires [8-10] are also being tested, since one microwire does not have the necessary amount of energy, but they have geometrical advantages compared to the ribbons. General dimensions of the sensor are: $80 \mathrm{~mm}$ in length, $10 \mathrm{~mm}$ in diameter including the plastic casing.

The magnetometer with this type of the sensor uses periodical saturation of the core caused by the current pulses to change its energetic state and to achieve steady initial conditions for the transient effects. If we consider a simplified linearized core model, the usable energy $\Delta W$ is given by the technical saturation $B_{s}$ from one side and by the variable energy value linearly dependent on the external magnetic field $B_{e x}$ on the other side, while $B_{e x}<B_{s}$ :

$$
\Delta W=\frac{V \mu_{e f}}{2 \mu_{0}}\left(\frac{B_{s}}{\mu_{e f}}-B_{e x}\right)^{2}
$$

where $V$ is the volume of the ferromagnetic core and $\mu_{e f}$ is the effective permeability of the core.

The energy $\Delta W$ is converted from the magnetic energy into the electrical energy by the sensing coil with the relaxation circuit. The energetic efficiency of this conversion is sufficient for processing without any high gain amplifiers. The electrical current $I_{0}$ flowing through the sensing coil right after the excitation pulse is the base mediated quantity for the transient effects and is independent of the load of the sensing winding (excluding open contacts state). Energy accumulated in the core is equal to the sensor energy and therefore we can write for the initial current $I_{0}$ : 

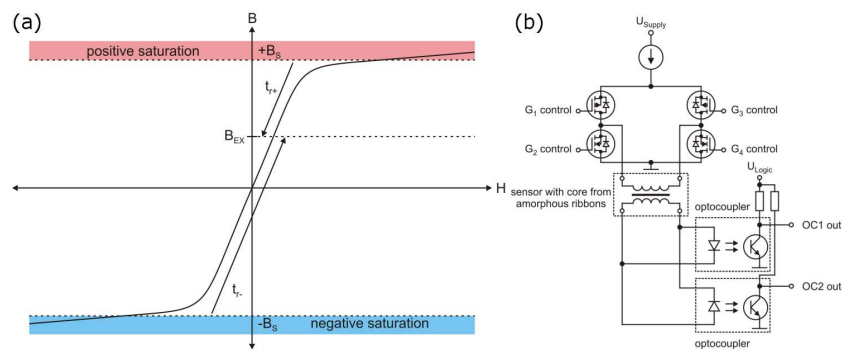

Fig. 1. Functional principle: (a) relaxation times creation, (b) scheme of realization.

$$
I_{0}=\frac{l}{n \mu_{0}}\left(\frac{B_{S}}{\mu_{e f}}-B_{e x}\right)
$$

where $n$ is the number of turns of the sensing winding and $l$ is the length of the sensing coil. Considering the beginning of the relaxation time interval in the time of the ideal falling edge of the excitation pulse, measurable transient effects occur on the terminals of the sensing coil with connected load. This gives three basic evaluation methods: sampling the voltage on the load, accumulation of the voltage on the load, and measurement of the transient effect duration conversion to time. The latter method is very efficient and relatively simple if the semiconductor diode is used to create the parametric load for $i_{D}>0$ :

$$
i_{D}=I_{0}-\frac{U_{T}}{L} t, \quad u_{D}=U_{T},
$$

where $U_{T}$ is the diode threshold voltage, $L$ is the inductance, and $t$ is time. Then for the relaxation time $t_{r}$, based on the linear non-resistant model of the circuit and the coil, we can write

$$
t_{r}=N S\left(B_{s}-\mu_{e f} B_{e x}\right) / U_{T},
$$

where $S$ is the transverse area of the core. The two diodes of the used 2-channel optocoupler are connected in anti-parallel (Fig. 1b). The measured magnetic field is proportional to the measured $t_{r+}$ and $t_{r-}$ time intervals

$$
B_{e x}=k\left(t_{r+}-t_{r-}\right) \text {. }
$$

\section{Measurement chain}

The magnetometer is designed as a modular system, which is achieved by dividing the magnetometer into the excitation module, sensing module, primary processing module, and secondary processing module. This allows to use the standard sensors as in the previous VEMA or HFT systems [1] but also to test new types of relaxation sensors. Also, relatively simple upgrading of the system can be performed since only the required module has to be changed if necessary.

The measurement chain of one channel of the relaxtype magnetometer with the direct optocoupler relaxation is shown in Fig. 2a. The relaxation circuits consist only from digital two-channel optocouplers with necessary passive components and the excitation circuitry
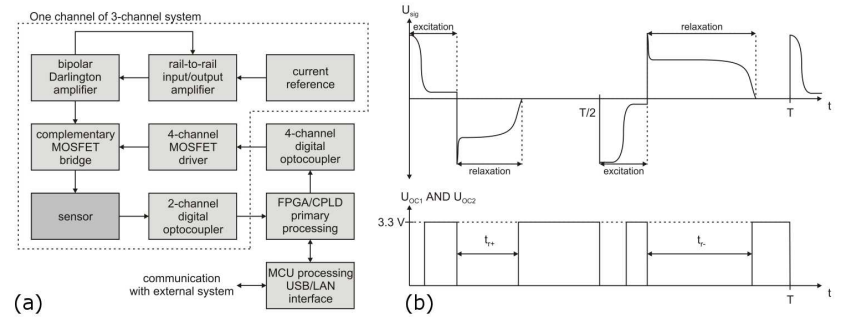

Fig. 2. Measurement with one of the magnetometer's channel: (a) block diagram of the chain, (b) illustration of the signals.

uses the full MOSFETs bridge powered from the current source. This gives a signal similar to the pulse-width modulation and can be effectively evaluated in digital circuits using the AND function between the optocouplers outputs OC1 and OC2 (Fig. 1b). The excitation control, the time intervals measurements, and the primary signal processing is carried out by the small FPGA/CPLD connected to the microcontroller for further signals processing and also to realize communication with supervising system like PC. With the use of the FPGA/CPLD parallel, simultaneous primary signal processing is achieved.

Using two unipolar power supplies and galvanic separation provided by the optocouplers the digital and analog grounds are separated which improves the magnetometer noise performance. Also only one unipolar power supply for the excitation circuitry improves the metrological properties of the system, since both, positive and negative, excitation signals are derived from one source.

\section{Measurement results}

The testing of the design was performed in standard laboratory conditions with the use of the Helmholtz coils, Agilent 34410A multimeter, Tektronix AFG1022 generator, GW Instek GPD-4303S DC source, which were all connected to the $\mathrm{PC}$ to create the programmable automated measurement workstation. Modules used during the testing included the FPGA MAX10 from Intel and the ARM Cortex-M7 microcontroller from ST Microelectronics. The static transfer characteristics of one measurement channel of the magnetometer with $1 \mathrm{kHz}$ sampling rate, $40 \mathrm{~mA} / 200 \mu$ s excitation pulses, and the counters clocked at $200 \mathrm{MHz}$ are shown in Fig. 3. The sum of time intervals can be additionally used for the thermal compensation of the measurement, since the temperature influences the $U_{T}$ of the relaxation diodes in both of the optocoupler channels equally and this dependence is practically linear. The tested sensor had the core made from 8 pcs of $2 \mathrm{~mm} \times 80 \mathrm{~mm}$ VAC6025 ribbons. Parameters of the inverse characteristics are summarized in Table I, where one LSB corresponds to 5 ns. The polynomial fit is applied only for $\pm 80 \mu \mathrm{T}$ range, since in smaller range the linear approximation is sufficient.

The linear spectral densities of the magnetometer channel without and with the core are shown in Fig. 4. 


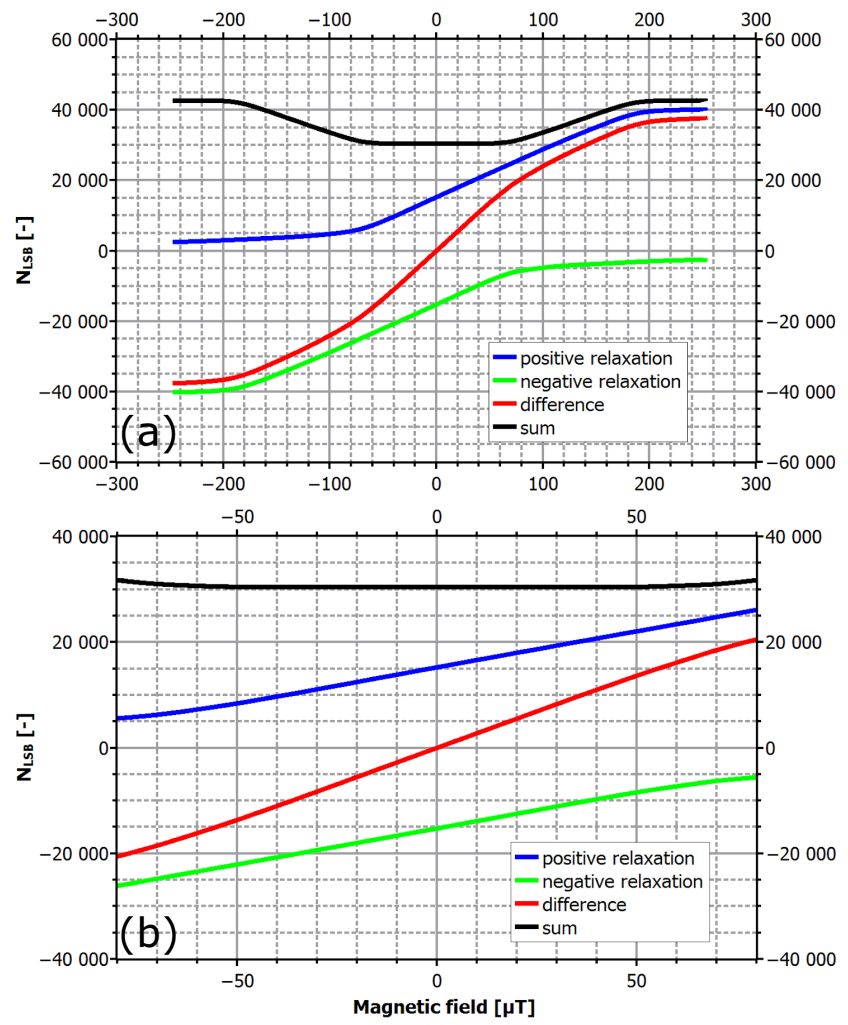

Fig. 3. Static transfer characteristics with tested sensor: (a) full measurement, (b) usable measurement area with acceptable $1 \%$ non-linearity detail.

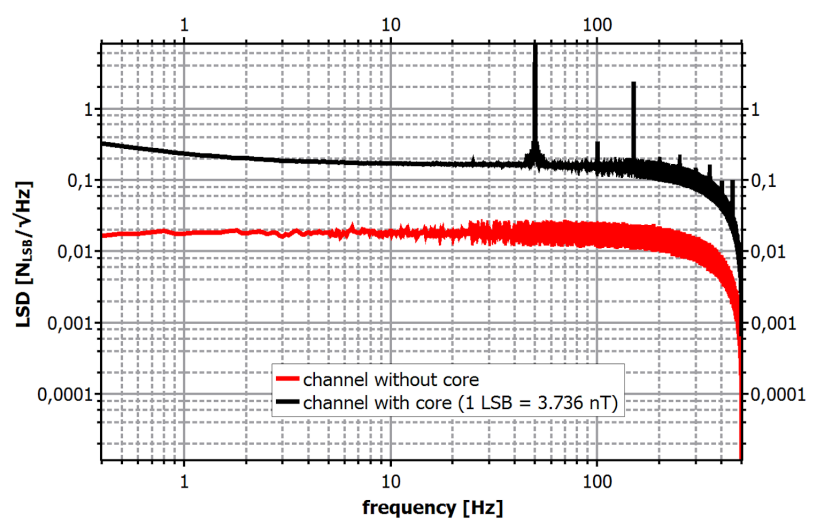

Fig. 4. Linear spectral densities of the magnetometer channel.

The measurements were performed in the ordinary laboratory conditions. Thus, the industrial frequencies from the power grid can be seen. With consideration of the transfer constant from Table I, the linear spectral densities are $800 \mathrm{pT} / \mathrm{Hz}^{1 / 2}$ at $1 \mathrm{~Hz}$ and $660 \mathrm{pT} / \mathrm{Hz}^{1 / 2}$ at $10 \mathrm{~Hz}$ for the tested magnetometer channel configuration.
TABLE I

Inverse characteristics approximations properties.

\begin{tabular}{|c|c|c|c|}
\hline \multicolumn{4}{|c|}{ Linear: $a x+b$} \\
\hline $\begin{array}{c}a \\
{[\mathrm{nT} / \mathrm{LSB}]}\end{array}$ & & $\begin{array}{c}b \\
{[\mathrm{nT}]}\end{array}$ & Non-linearity \\
\hline 3.736 & & 3.945 & $\begin{array}{l}\leq 1 \% \mathrm{FS} \text { in } \pm 80 \mu \mathrm{T} \\
\leq 0.3 \% \mathrm{FS} \text { in } \pm 60 \mu \mathrm{T}\end{array}$ \\
\hline \multicolumn{4}{|c|}{ Polynomial: $a x^{3}+b x+c$} \\
\hline $\begin{array}{c}a \\
{\left[\mathrm{nT}^{2} \mathrm{LSB}^{3}\right]}\end{array}$ & $\begin{array}{c}b \\
{[\mathrm{nT} / \mathrm{LSB}]}\end{array}$ & $\begin{array}{c}c \\
{[\mathrm{nT}]}\end{array}$ & $\begin{array}{c}\text { Max. error } \\
\text { (incl. background) }\end{array}$ \\
\hline $7.352 \times 10^{-13}$ & 3.542 & -23.945 & $0.3 \% \mathrm{FS}$ in $\pm 80 \mu \mathrm{T}$ \\
\hline
\end{tabular}

\section{Conclusions}

The testing results show that although the presented design is relatively cheap and simple, the achievable sensitivity and noise parameters are sufficient for a wide range of applications in the industry like ferromagnetic objects detection, contactless diagnostics of electrical devices, magnetic field monitoring, and also for educational purposes, since the sensors are not fixed together, but can be placed in arbitrary positions and directions.

\section{Acknowledgments}

This work was supported by the APVV-17-0184, APVV-18-0248, VEGA No. 1/0374/17, KEGA No. 052TUKE-4/2018, and KEGA No. 058TUKE$4 / 2018$ projects.

\section{References}

[1] D. Praslička, J. Blažek, J. Hudák, I. Mikita, V. Moucha, JEEEC 66, 190 (2015).

[2] P. Ripka, Magnetic Sensors and Magnetometers, Artech House Publ., 2001.

[3] S. Tumanski, Handbook of Magnetic Measurements, CRC Press, 2011.

[4] V. Korepanov, A. Marusenkov, Surv. Geophys. 33, 1059 (2012).

[5] J. Blažek, J. Hudák, D. Praslička, Sens. Actuat. A 59, 287 (1997).

[6] D. Praslička, I. Mikita, Int. J. Appl. Electromagn. Mech. 25, 489 (2007).

[7] J. Marcin, A. Klinda, P. Švec, D. Praslicka, J. Blažek, J. Kováč, P. Svac Sr., I. Škorvánek, IEEE Trans. Magn. 46, 416 (2010).

[8] K. Richter, R. Varga, A. Thiaville, IEEE Trans. Magn. 50, 2501404 (2014).

[9] P. Klein, K. Richter, R. Varga, M. Vazquez, J. Alloys Comp. 569, 569 (2013).

[10] K. Richter, R. Varga, J. Kovac, A. Zhukov, IEEE Trans. Magn. 48, 1266 (2012). 